\title{
RF SYSTEM FOR THE SLS BOOSTER AND STORAGE RING
}

\author{
M.E. Busse-Grawitz, P. Marchand, W. Tron, Paul Scherrer Institute, CH 5232 Villigen PSI
}

\section{Abstract}

The RF system which was adopted for the SLS (Swiss Light Source) initial phase [1] is based on the use of conventional, already well-proven equipment. In the storage ring (SR), it consists of four $500 \mathrm{MHz}$ plants, each comprising a normal conducting (nc) single-cell cavity of the ELETTRA type, powered with a $180 \mathrm{~kW} \mathrm{CW} \mathrm{klystron}$ amplifier via a WR1800 waveguide line. In spite of the lower power requirement, one similar plant will be used for the booster with the intention of standardizing. Although the use of superconducting (sc) cavities has been ruled out as a starting solution, combining idle (no external RF source) sc cavities with the initial nc system is regarded as a possible way of further improving the beam lifetime in the SR when operating at very high brightness [2].

\section{MAIN DESIGN PARAMETERS}

The basic parameters of the SR which were determining for the design of the RF system are listed in Table 1. At the nominal energy of $2.4 \mathrm{GeV}$ and beam current of $0.4 \mathrm{~A}$, the radiation power to be restored by the RF system is $240 \mathrm{~kW}$. A peak accelerating voltage of $2.4 \mathrm{MV}$ (at $500 \mathrm{MHz}$ ) is needed to achieve the energy acceptance of $\pm 3.5 \%$.

Table 1 : Basic SR parameters

\begin{tabular}{|l|c|}
\hline Circumference $\mathrm{L}[\mathrm{m}]$ & 288. \\
Revolution frequency, $\mathrm{f}_{\mathrm{o}}[\mathrm{MHz}]$ & 1.04 \\
Energy, $\mathrm{E}[\mathrm{GeV}]$ & 2.4 \\
Radiation loss / turn, $\Delta \mathrm{U}[\mathrm{MeV}]$ & 0.6 \\
Beam current, $\mathrm{I}_{\mathrm{b}}[\mathrm{A}]$ & 0.4 \\
Beam power loss, $\mathrm{P}_{\mathrm{b}}[\mathrm{kW}]$ & 240. \\
Momentum compaction, $\alpha$ & $7 . \mathrm{E}-4$ \\
Momentum spread, $\sigma_{\mathrm{p}}[\%]$ & 0.09 \\
Longitudinal damping time $\tau_{\mathrm{s}}[\mathrm{ms}]$ & 4.5 \\
Transverse damping time, $\tau_{\mathrm{x}, \mathrm{y}}[\mathrm{ms}]$ & 9. \\
RF frequency, $\mathrm{f}_{\mathrm{RF}}[\mathrm{MHz}]$ & 499.652 \\
Harmonic number, $\mathrm{h}=\mathrm{f}_{\mathrm{RF}} / \mathrm{f}_{\mathrm{o}}$ & 480 \\
RF voltage, $\mathrm{V}_{\mathrm{RF}}[\mathrm{MV}]$ & 2.4 \\
RF acceptance, $\varepsilon_{\mathrm{RF}}[\%]$ & \pm 3.5 \\
Bunch length, $\sigma_{\mathrm{s}}[\mathrm{mm}]$ & 4. \\
Synchrotron frequency, $\mathrm{f}_{\mathrm{s}}[\mathrm{kHz}]$ & 7.5 \\
Synchronous phase, $\phi_{\mathrm{s}}[\mathrm{degree}]$ & 14.5 \\
\hline
\end{tabular}

\section{RF CAVITY ASSEMBLY}

Four nc single-cell cavities of the ELETTRA type [3] with a shunt impedance of $3.4 \mathrm{M} \Omega$ and a quality factor of 40000 will provide the required RF voltage and power in the SR. The parameter values in Table 2 show that, using four such cavities, the RF system has the potential for achieving the SLS requirement with a cavity input power $\left(\mathrm{P}_{\mathrm{t}} / \mathrm{cav}\right)$ lower than $150 \mathrm{~kW}$. Besides, would one of the four cavities be out of use, the operation at full beam current is still possible with an RF voltage of about $2 \mathrm{MV}$. The four cavities will be accommodated in pairs in two of the dispersion free, low $\beta, 4 \mathrm{~m}$ long straight sections of the SR.

The input coupler must be capable to feed into the cavity a CW RF power of at least $150 \mathrm{~kW}$ (forward) and also to handle the full reflection. It will be similar to those operating in ELETTRA which are of the coaxial type, terminated by a coupling loop. Although the operating power at ELETTRA does not exceed $65 \mathrm{~kW}$, the same coupler has successfully been tested up to $330 \mathrm{~kW} \mathrm{[4]} \mathrm{and}$ therefore should be capable to fulfil the SLS requirement. The coupling coefficient shall be adjustable within a range of 1 to 3.3 in order to match different beam loading conditions.

The cooling system consists of water flowing through pipes which are brazed on the cavity outer surface and must be able to remove up to $65 \mathrm{~kW}$ of power dissipation into the cavity wall $\left(\mathrm{P}_{\mathrm{d}} / \mathrm{cav}\right)$. In addition, it shall be possible - thanks to the cooling system - to set the cavity operating temperature at any value within $60 \pm 25^{\circ} \mathrm{C}$ with a stability of $\pm 0.05{ }^{\circ} \mathrm{C}$. This is achieved by properly isolating the cavity and by re-circulating the cooling water through an appropriate heat exchanger water station (cooling rack) dedicated to each cavity. Controlling the cavity temperature - and therefore the HOM frequencies in a way to avoid resonance excitations by the beam should allow to prevent coupled bunch instabilities $[1,5]$.

A plunger tuner provides an additional degree of freedom in tuning the fundamental and HOM's.

The tuning of the fundamental frequency for variable beam loading - with different operating temperatures and plunger positions - will be performed by means of a mechanical system driven with a stepping motor which will change the cavity length (longitudinal squeezing or stretching), within the range of elastic deformation. In operation, the cavities will be automatically tuned by a regulation loop (see section 4 ).

Table 2 : RF operating parameters with 4 and $3 / 4$ cav. $\left(\beta_{\mathrm{m}}, \Delta \mathrm{f}_{\mathrm{m}}\right.$ : optimum coupling factor and detuning)

\begin{tabular}{|c|c|c|c|c|c|c|c|}
\hline $\begin{array}{c}\mathrm{I}_{\mathrm{b}} \\
{[\mathrm{A}]}\end{array}$ & $\begin{array}{c}\mathrm{V}_{\mathrm{RF}} \\
{[\mathrm{MV}]}\end{array}$ & $\begin{array}{c}\mathrm{P}_{\mathrm{d}} / \mathrm{cav} \\
{[\mathrm{kW}]}\end{array}$ & $\begin{array}{c}\mathrm{P}_{\mathrm{b}} / \mathrm{cav} \\
{[\mathrm{kW}]}\end{array}$ & $\begin{array}{c}\mathrm{P}_{\mathrm{t}} \\
/ \mathrm{cav} \\
{[\mathrm{kW}]}\end{array}$ & $\beta_{\mathrm{m}}$ & $\begin{array}{c}\Delta \mathrm{f}_{\mathrm{m}} \\
{[\mathrm{kHz}]}\end{array}$ & $\begin{array}{c}\mathrm{nb} \text { of } \\
\text { cav. }\end{array}$ \\
\hline 0.4 & 2.6 & 60. & 60. & 120. & 2.0 & 26. & 4 \\
0.4 & 2.0 & 65. & 80. & 145. & 2.2 & 24. & 3 \\
\hline
\end{tabular}




\section{RF AMPLIFIER AND FEEDER LINE}

Each cavity will be individually powered with a CW klystron amplifier capable to deliver more than $180 \mathrm{~kW}$ (efficiency $>60 \%$ ). The klystrons will be provided by EEV Ltd, the DC power supplies for the cathode and auxiliaries (anode modulation, cathode heating, focal coils, ion pump) by THOMCAST AG.

The DC power supply for the cathode is a Pulse Step Modulator (PSM), a technology currently used for broadcast transmitters [6]. Designed for $46 \mathrm{kV}-7.5 \mathrm{~A}$, this PSM essentially consists of 68 power modules which are connected in series and supplied through their own secondary winding from two transformers. The two transformers are shifted in phase, resulting in a 12-pulse loading of the mains with a 6-pulse rectification in the module chain. Each one of the 68 modules represents an autonomous voltage source (Us $\approx 800 \mathrm{~V}$ ) which may be switched on/off individually by means of fast IGBT switches operating up to $14 \mathrm{kHz}$. The switching sequence and pulse duration is generated and supervised by the PSM control system such that the thermal loading of all modules is distributed equally. The switching frequency can be suppressed at the output of the module chain, by means of a low pass filter.

The main PSM features (efficiency, regulation speed and accuracy, compatibility with large variation of the load impedance) are well suited for our purpose. Moreover, the modular concept with high redundancy (up to four defective modules without performance degradation) makes it very reliable, easy to maintain and there is no need for HV crowbars.

The RF power delivered by the klystron is fed into the cavity input coupler via a WR1800 waveguide line including monitoring directional couplers as well as a circulator to isolate the klystron from the variable (beamloaded) cavity impedance. All these waveguide components are commercially available. The four RF plants of the SR are arranged in pairs in two diagonally opposite RF stations. Figure 1 shows a layout of one of them.

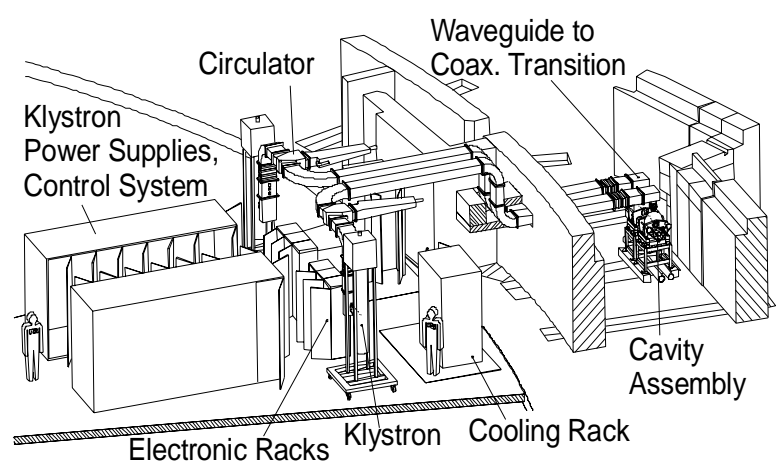

Figure 1 : Layout of one of the two SR RF stations

\section{LOW LEVEL ELECTRONIC SYSTEM}

The low level electronic system comprising standard frequency, amplitude and phase regulation loops as well as the amplifier drive chain and the RF signal measurement channels will be supplied by Sincrotrone Trieste. It will be based on the system operating in ELETTRA with minor modifications for the SLS purpose as described below. A simplified block diagram is shown in Figure 2.

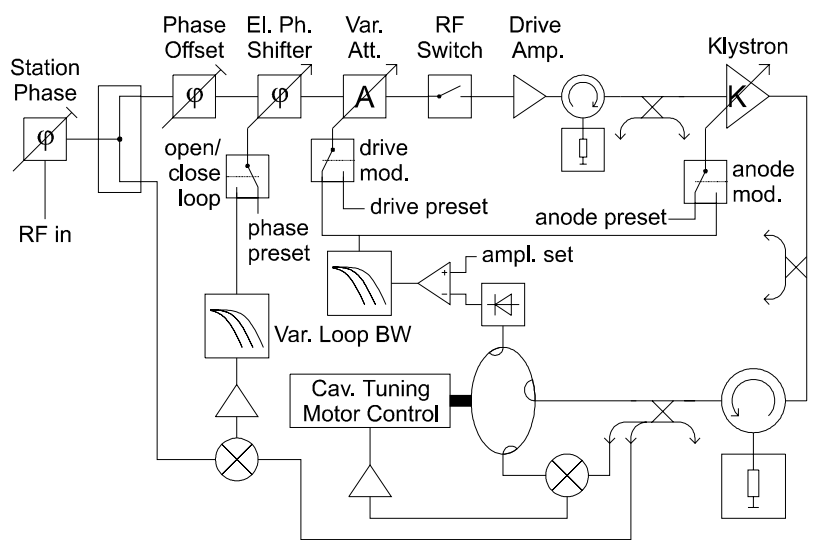

Figure 2 : Low level electronic system

The frequency regulation loop has a tuning range of $\pm 200 \mathrm{kHz}$ which corresponds to a change in cavity length of $\pm 0.2 \mathrm{~mm}$ (within the limit of elastic deformation). This allows to handle cavity temperature variations of about $\pm 20^{\circ} \mathrm{C}$ with a sufficient margin for the compensation of the largest beam loading effect which is around $25 \mathrm{kHz}$ (see Table 2). The maximum tuning speed of $1 \mathrm{kHz} / \mathrm{s}$ is fast enough for injecting the full beam in less than $30 \mathrm{~s}$ and the sensitivity of the loop (adjustable between $\pm 100 \mathrm{~Hz}$ and $\pm 1 \mathrm{kHz}$ ) will be experimentally optimised such that to avoid undue wear of the tuner.

The amplitude loop whose $3 \mathrm{~dB}$ bandwidth is adjustable up to $5 \mathrm{kHz}$ regulates the cavity accelerating voltage with a stability better than $1 \%$ by controlling the drive power. The option for modulating the klystron anode voltage - instead of the drive power - is implemented as an alternative scheme of amplitude regulation.

The phase loop compensates for the phase changes (up to $\pm 30^{\circ}$ ) in the amplification chain with variable power. It also has a $3 \mathrm{~dB}$ bandwidth adjustable up to $5 \mathrm{kHz}$ and must ensure a phase stability of $\pm 0.5^{\circ}$.

The bandwidth of both the phase and amplitude loops will be experimentally optimized to provide enough damping while remaining insensitive to the synchrotron frequency.

In the drive chain the signal from the $500 \mathrm{MHz}$ master oscillator, after being split, phase and amplitude regulated, is amplified with a $50 \mathrm{~W}$ solid state amplifier. A fast RF switch at the input of the chain can remove within less than $5 \mu$ s the driving RF signal under certain conditions (beam dump, klystron/cavity interlocks). 


\section{RF CONTROL SYSTEM}

The RF control system will be produced in collaboration between THOMCAST and PSI. A block diagram is shown in Figure 3.

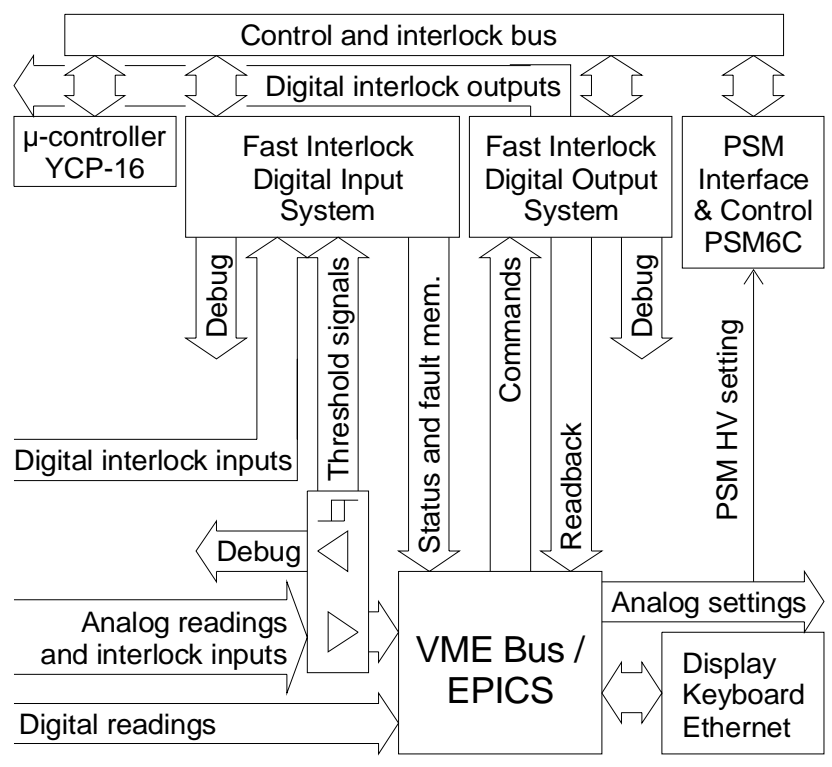

Figure 3 : Block diagram of the RF control system

The top level controller consists of a VME-bus crate operating under VxWorks and EPICS with an associated keypad and visual display. This constitutes the operatormachine interface.

The control of the PSM is ensured by the PSM6C unit, a THOMCAST standard control and interface system which communicates with the PSM modules via fibre optic links.

The interlock protection system consists of a fast microcontroller (YCP16 from THOMCAST) and digital input/output printed circuit boards with field programmable logic devices. The interlock state machine which controls the start-up and switch-off sequences of the various RF equipment parts is realised in YCP16 and each digital interlock input/ output signal has its own logic programmed locally. In this logic, it is programmed under which condition the input has to cause a fallback of the state machine and the output (action) is processed as a function of the actual state and commands from EPICS. The analog interlock inputs are converted into digital inputs by means of comparators with adjustable thresholds. For each interlock input, three memories are used which store the first fault event, the status of all the signals at that time and the sequence of the faults occurring afterwards (up to 9 events). The fault event and status information is available in EPICS and also indicated on the front panel of the printed circuit boards by means of two-colour LED's.

The different sub-units communicate via the control and interlock bus. Fibre optical links are used for all signals at high potential and the other input/ output signals are galvanically isolated by means of opto-couplers or isolating amplifiers. The relevant analog and digital signals are also available on a connector panel for debugging purposes.

This system will be integrated in the SLS central control system - also based on VME and EPICS - for remote operation from the control room; nevertheless, it shall allow a stand-alone operation of each RF plant (independently of the SLS central system).

\section{BOOSTER RF SYSTEM}

A single cavity can provide the maximum RF voltage of $0.5 \mathrm{MV}$ required for the booster. With the beam current of $12 \mathrm{~mA}$, the cavity input power of $40 \mathrm{~kW}$ is significantly less than in the SR. However, with the intention of standardising we decided to use one $500 \mathrm{MHz}$ plant similar to those of the SR. The major difference is that the RF voltage shall be cycled at $3 \mathrm{~Hz}$ by varying the reference voltage of the amplitude regulation loop as a function of the magnet field program. Contrary to the SR, the coupled bunch instabilities should not be an issue and therefore the cooling rack for the temperature control of the HOM frequencies is not needed.

\section{POSSIBLE FURTHER UPGRADING}

The RF system described before - although quite conventional and while operating at relatively conservative performance levels - should be capable to achieve the SLS nominal requirements.

The use of sc cavities has been ruled out as a starting solution. However, for improving the beam lifetime in the SR when operating at very high brightness, the initial nc system could be further complemented with idle (beamdriven) sc cavities. Within this scheme, the nc system provides the power to the beam and the sc system only contributes to the potential well. Two possible applications of such a system were considered: either doubling the fundamental RF voltage with one $500 \mathrm{MHz}$ sc cavity, or lengthening the bunches - by a factor of about four - with one (possibly two) third harmonic sc cavity(ies) [2]. Computer simulations indicated that the latter option would be more efficient for our purpose [7] and this is therefore the approach presently investigated.

\section{REFERENCES}

[1] P. Marchand, PSI Note SLS-TME-TA-1998-0011.

[2] P. Marchand, PSI Note SLS-TME-TA-1998-0012 and these Proc.

[3] M. Svandrlik et al EPAC98, p. 1817 - 1819, 1998.

[4] M. Svandrlik, private communication.

[5] M. Svandrlik et al, PAC97, p. 1735 - 1737, 1997.

[6] THOMCAST AG, TSM6 - E.PM5, Sept. 1996.

[7] M. Boege et al, these Proceedings 